\title{
Modelling nonlinear viscoelastic behaviours of loudspeaker suspensions-like structures
}

\author{
Balbine Maillou, Pierrick Lotton, Antonin Novak*, Laurent Simon \\ Laboratoire d'Acoustique de I'Université du Mans, LAUM - UMR 6613 CNRS, Le Mans Université, Avenue Olivier \\ Messiaen, 72085 LE MANS CEDEX 9, France \\ *antonin.novak@univ-lemans.fr \\ *https://ant-novak. com
}

\begin{abstract}
Mechanical properties of an electrodynamic loudspeaker are mainly determined by its suspensions (surround and spider) that behave nonlinearly and typically exhibit frequency dependent viscoelastic properties such as creep effect. The paper aims at characterizing the mechanical behaviour of electrodynamic loudspeaker suspensions at low frequencies using nonlinear identification techniques developed in recent years. A Generalized Hammerstein based model can take into account both frequency dependency and nonlinear properties. As shown in the paper, the model generalizes existing nonlinear or viscoelastic models commonly used for loudspeaker modelling. It is further experimentally shown that a possible input-dependent law may play a key role in suspension characterization.
\end{abstract}

The archived file is not the final published version of the article B. Maillou, P. Lotton, A. Novak \& L. Simon (2018), "Modelling nonlinear viscoelastic behaviours of loudspeaker suspensions-like structures", Journal of Sound and Vibration. Vol. 416(0), pp. 213-223.

The definitive publisher-authenticated version is available online at https://doi.org/10.1016/j.jsv.2017.11.046, Readers must contact the publisher for reprint or permission to use the material in any form. 


\section{Introduction}

Suspensions (surround and spider) play an important role in electrodynamic loudspeaker design and operation. Their role is twofold : first to centre and adjust the voice-coil in the magnetic air gap, allowing an axial motion of the diaphragm while preventing lateral motion or rocking ; second to provide the restoring force. The materials used together with the assembly geometry usually result in a complex nonlinear viscoelastic behaviour, even at low amplitude of excitation.

On the one hand, there are many studies dealing with mechanical systems and their nonlinear dynamics including viscoelastic properties $[1,2,3]$ that take into account many nonlinear phenomena, temperature dependence $[4,5]$ or even time-variation [6] of the viscoelastic materials. On the other hand, in the today's most used classical model [7], the moving part of a loudspeaker is usually described by a simple mass-spring-damper linear system. Besides, more accurate linear descriptions have been proposed, taking into account the frequency dependence of damping and/or stiffness due to viscoelasticity $[8,9]$. Since there are many nonlinear phenomena including the viscoelastic effects of the suspensions [10], the classical linear model is not sufficient for describing the loudspeaker behaviour for larger amplitudes. In order to describe at least partly these phenomena, the stiffness of the mass-spring damper model is usually described as a nonlinear function of instantaneous displacement expressed in a polynomial way [10].

Even if the viscoelastic and nonlinear behaviours are known for decades [11], none of the existing models of loudspeaker suspensions take simultaneously both effects (frequency dependence of damping and/or stiffness and nonlinear effects) into account. In this paper, we propose a nonlinear model of the moving part of an electrodynamic loudspeaker taking into account both the nonlinear behaviour together with the complex viscoelastic phenomena.

The structure of the proposed model, derived from the basis of existing viscoelastic and nonlinear models in section 2, is shown to be very similar to the structure of the so called Generalized Hammerstein model with polynomial inputs (section 3) that has been successfully used to model nonlinearities in other fields of physics $[12,13,14]$ and for which several measurement techniques have been developed in recent years [15, 16, 17]. To apply one of these techniques for the study of dynamical behaviour of loudspeaker suspensions, we propose an experimental bench (section 4) in which the mechanical part of the loudspeaker is separated from the loudspeaker to be measured apart. The experimental results from the measurement on an off-the-shelf loudspeaker are presented in section 6 and a discussion of the main important results is proposed in section 6 with a concluding summary.

\section{State of the art}

In its simplest form, the behaviour of the moving part of an electrodynamic loudspeaker is described by a massspring-damper equation

$$
M_{m s} \frac{\mathrm{d}^{2} x(t)}{\mathrm{d} t^{2}}+R_{m s} \frac{\mathrm{d} x(t)}{\mathrm{d} t}+K_{m s} x(t)=F(t),
$$

with $M_{m s}$ the mass of the diaphragm, $R_{m s}$ and $K_{m s}$ the damping (also called mechanical resistance) and the stiffness of the suspension, respectively, $F(t)$ the force created by the current passing through the voice-coil, and $x(t)$ the displacement of the moving part (considering only a piston motion). The relation (1) suffers from two drawbacks: first it is valid only for small displacements corresponding to linear behaviour and, second, it does not take into account the viscoelastic properties of the suspensions. Both drawbacks and existing solutions are briefly described below in the remaining of this section.

As shown in $[10,18,19,20,21]$, expression (1) does not succeed in describing the nonlinear behaviour in the large signal domain. Indeed, in case of high input level, the stiffness factor $K_{m s}$ can no longer be considered as constantvalued but varies with the displacement $x(t)$. This behaviour can be modelled using polynomial approximations [10, 22, 23, 24]. Besides, the damping $R_{m s}$ is usually considered independent of displacement or velocity. However, 
as suggested in [25] and later demonstrated in [26], the damping $R_{m s}$ can also vary with instantaneous velocity and/or displacement [27, 28].

Considering here both the damping $R_{m s}$ and the stiffness $K_{m s}$ nonlinearly depending on the instantaneous displacement $x(t)$, we can modify the expression (1) in the following manner

$$
M_{m s} \frac{\mathrm{d}^{2} x(t)}{\mathrm{d} t^{2}}+\left(\sum_{n=1}^{N} r_{n} \cdot x^{n-1}(t)\right) \frac{\mathrm{d} x(t)}{\mathrm{d} t}+\left(\sum_{n=1}^{N} k_{n} \cdot x^{n-1}(t)\right) x(t)=F(t),
$$

$N \in \mathbb{N}^{*}$ being the model order, and the parameters $r_{n}$ and $k_{n}$ being the coefficients of the polynomial expansion respectively for the damping and the stiffness.

As shown in $[8,9,29,30]$, the linear mass-spring-damper equation (1) has a limited use even in small signal domain. Indeed, materials used for loudspeaker suspensions exhibit viscoelastic properties leading to behaviours that are far more complex than those described with a simple mass-spring-damper representation. In [8], several linear models considering frequency dependent parameters are described, the most accurate one being the so-called LOG (logarithmic) model. In [31], fractional derivatives are successfully used to model the viscoelastic behaviour. All these models show that either an extra parameter must be added to the mass-spring-damper system, or frequency dependent parameters $K_{m s}$ and $R_{m s}$ must be considered.

The time domain relation between force and displacement from Eq. (1) can be expressed in the frequency domain including the frequency dependent parameters $K_{m s}(f)$ and $R_{m s}(f)$ as (with $\mathrm{i}=\sqrt{-1}$ )

$$
-(2 \pi f)^{2} M_{m s} X(f)+\mathrm{i} 2 \pi f R_{m s}(f) X(f)+K_{m s}(f) X(f)=F(f) .
$$

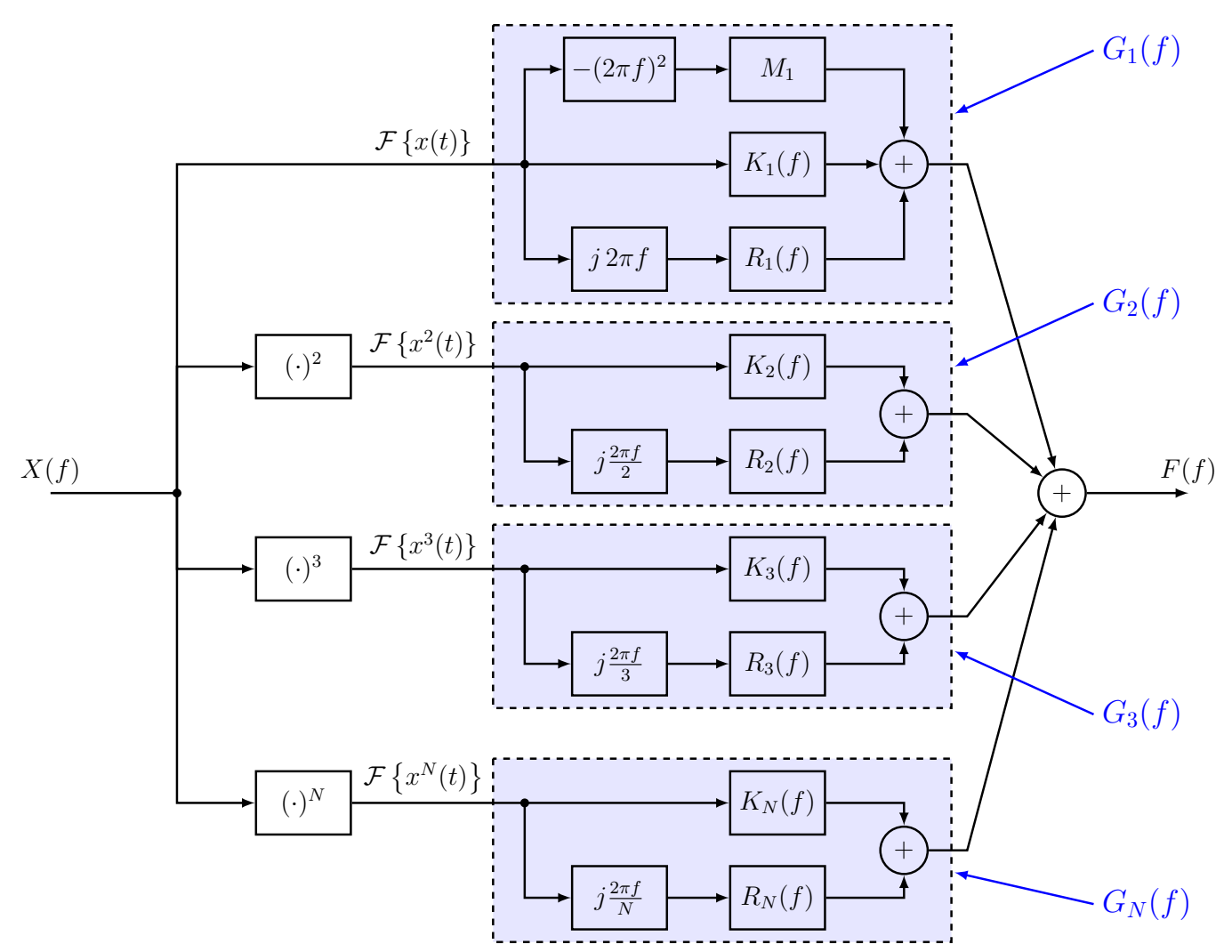

Figure 1. Block diagram representation of the moving part loudspeaker including stiffness $K_{n}(f)$ and damping $R_{n}(f)$. 
In order to consider both effects presented in this section (the instantaneous displacement dependence and the frequency dependence of both damping and stiffness) a so-called viscoelastic nonlinear model is proposed in the next section.

\section{Visco-elastic nonlinear model}

The model proposed in this paper takes into account the frequency dependence of stiffness and damping from Eq. (3) together with the nonlinear functions represented in time domain in Eq. (2), we either have to express the frequency dependence of parameters $K_{m s}$ and $R_{m s}$ in time domain using a convolution or to express the nonlinear laws in frequency domain. Both solutions being equivalent from the mathematical point of view, we choose to use the frequency domain for the sake of simplicity.

\section{Considering}

$$
X^{(n)}(f)=\mathcal{F}\left\{x^{n}(t)\right\}
$$

and

$$
\mathcal{F}\left\{x^{n-1}(t) \frac{\mathrm{d} x(t)}{\mathrm{d} t}\right\}=\mathcal{F}\left\{\frac{1}{n} \frac{\mathrm{d} x^{n}(t)}{\mathrm{d} t}\right\}=\mathrm{i} \frac{2 \pi f}{n} X^{(n)}(f),
$$

$\mathcal{F}$ representing the Fourier transform, we propose to express the nonlinear frequency-dependent behaviour in a polynomial way as

$$
-M_{m s}(2 \pi f)^{2} X(f)+\sum_{n=1}^{N} X^{(n)}(f) \cdot\left[K_{n}(f)+\mathrm{i} \frac{2 \pi f}{n} \cdot R_{n}(f)\right]=F(f) .
$$

This relation, describing the nonlinear behaviour of suspensions, is a generalized form of both equations (2) and (3), and can be represented by a block diagram as depicted in Fig. 1. Note, that this schematic representation is very similar to a Generalized Hammerstein structure made up of $N$ parallel branches, each branch consisting of a linear filter $G_{n}(f)$ preceded by a $n$th power static nonlinear function. Rewriting the expression (6) into the form of a Generalized Hammerstein model yields

$$
\sum_{n=1}^{N} X^{(n)}(f) \cdot G_{n}(f)=F(f)
$$

where the kernels $G_{n}(f)$ of the Generalized Hammerstein model are related to the frequency dependent coefficients $K_{n}(f)$ and $R_{n}(f)$ as

$$
K_{n}(f)=\left\{\begin{array}{cc}
\operatorname{Re}\left\{G_{n}(f)\right\}+M_{m s}(2 \pi f)^{2} & \text { for } n=1 \\
\operatorname{Re}\left\{G_{n}(f)\right\} & \text { for } n>1
\end{array}\right.
$$

and

$$
R_{n}(f)=\frac{n}{2 \pi f} \operatorname{Im}\left\{G_{n}(f)\right\} \quad \forall n .
$$

Note that the models from Eqs. (1-3) are subclasses of the proposed model from Eqs. (7-9). Taking $K_{n \geq 2}(f)=0$ and $R_{n \geq 2}(f)=0$ eliminates all the nonlinear effects of the model and results in Eq. (3). Considering $K_{n}(f)$ and $R_{n}(f)$ as constant-valued functions we get rid of frequency dependent parameters which leads to Eq. (2). Finally, Eq. (1), in which neither the nonlinear effect nor the frequency dependence is included, arises when both previous conditions are satisfied.

Consequently, the Generalized Hammerstein model presented in Eqs. (7-9) is a generalized case of both nonlinear and frequency dependent models. Hence, we consider in the following, that the mechanical part of the loudspeaker can be represented by a Generalized Hammerstein model for which identification techniques have been recently developed $[15,16]$. This model is tested in next sections on an off-the-shelf loudspeaker using an experimental set-up and a measurement procedure focused on the identification of the filters $G_{n}(f)$ and consequently on the estimation of $K_{n}(f)$ and $R_{n}(f)$. 


\section{Experimental set-up}

The experimental set-up developed and used to study the dynamical behaviour of loudspeaker suspensions is shown in Fig. 2. The magnetic motor is removed from the loudspeaker and the basket of the loudspeaker is fixed to a rigid mechanical structure. The moving part, consisting of the diaphragm, the voice-coil, the surround and the spider, is then mechanically excited by a shaker (type $L D S$ V406). The shaker is coupled to the voice-coil support using a rigid pushing rod. The force $F(t)$ applied to the moving part of the loudspeaker is measured using an impedance head (type $B \xi K$ 8001) set between the pushing rod and the voice-coil support. A laser sensor (type Panasonic LM10 ANR1282) is focused on the centre of the diaphragm to measure the displacement $x(t)$ of the moving part. An OROS analyser, driven by $N V$ Gate software, is used to acquire the sensors signals and generate the input signals supplied to the power amplifier $L D S$ PA100E connected to the shaker.

This bench is used to identify the unknown filters $G_{n}(f)$ from the known input and output signals $x(t)$ and $F(t)$ respectively. For this purpose, sinusoidal input voltage, controlled in both amplitude and frequency, is supplied to the power amplifier. Since the nonlinear behaviour of suspensions as well as their possible frequency dependence are known to be most important at low frequencies [8, 25], the frequency range is chosen from $250 \mathrm{mHz}$ up to $40 \mathrm{~Hz}$. Five input levels are tested corresponding to a maximum displacement $x_{\max }$ of the diaphragm from $0.2 \mathrm{~mm}$ up to $3 \mathrm{~mm}$. As the shaker response is not flat with frequency, the input voltage has to be adjusted to impose the same displacement $x_{\max }$ for each frequency.

Moreover, as the shaker is a nonlinear device, the shaker and the moving part under test can be seen as two coupled nonlinear systems (NLS) connected in series. Consequently, the unwanted nonlinearities of the shaker are mixed with the nonlinearities caused by the moving part. Recently, Novak et al. [32] developed a method allowing the identification of the two NLS in series in term of $N$ th-order Generalized Hammerstein model (see A.1 for more details). We use this method here to estimate the filters $G_{n}(f)$ corresponding to the moving part of the loudspeaker, getting rid of the nonlinear behaviour of the shaker.

The device under test for this paper is the moving part of a 3.3 inches Visaton Sc8n loudspeaker with a membrane made of paper, a surround made of filled rubber and a spider made of impregnated fibre. The data sheet indicates that its maximal elongation is $\pm 3 \mathrm{~mm}$.

The following three contributions must be taken into account while evaluating the total mass $M_{m s}: 1$ ) the mass of the loudspeaker moving part (membrane and voice-coil) estimated by the Klippel analyser [33] before removing the motor $(1.55 \mathrm{~g}), 2)$ the mass of an adaptive aluminium piece inserted between the impedance head and the moving part of the loudspeaker $(9.25 \mathrm{~g})$, and 3$)$ the mass below force gauge of the impedance head $(2.1 \mathrm{~g})$. The sum of these three contributions gives the total mass $M_{m s}=12.9 \mathrm{~g}$.

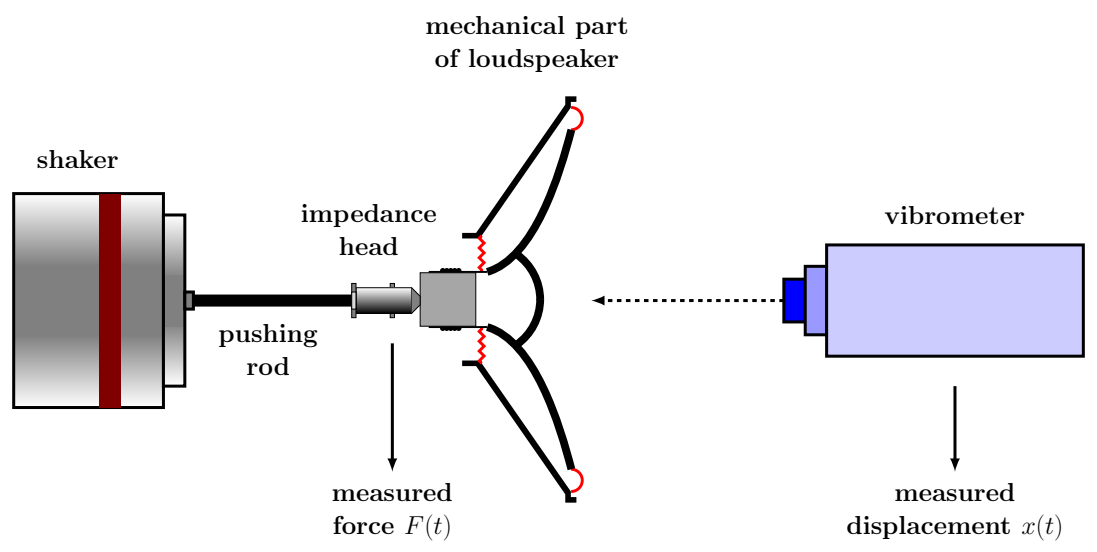

Figure 2. Experimental setup for testing the loudspeaker moving part mechanical behaviour, excited with a shaker. 


\section{Experimental results}

\subsection{Estimation of filters $G_{n}(f)$}

Both displacement and force signals are recorded for input frequency $f_{0} \in[250 \mathrm{mHz}-40 \mathrm{~Hz}]$ and for maximum input displacement of the diaphragm $x_{\max } \in[0.2 \mathrm{~mm}-3 \mathrm{~mm}]$.

These signals are then used as input (displacement) and output (force) of the Generalized Hammerstein model described by the relation (7). They allow the estimation of the filters $G_{n}(f)$ for $1 \leq n \leq N$, for each input level corresponding to $x_{\max }$.

As an example, Fig. 3 shows the modulus $\left|G_{1}(f)\right|$ as a function of frequency, estimated for each tested $x_{\max }$ value. Note that for this estimation, the order of the Hammerstein model is chosen to be $N=7$. The resonant frequency corresponding to the minimal value of $\left|G_{1}(f)\right|$ decreases with increasing input level. Since the filter $G_{1}(f)$ varies with the input level, we conclude that the moving part of a loudspeaker cannot be described by a unique input-independent Generalized Hammerstein model.

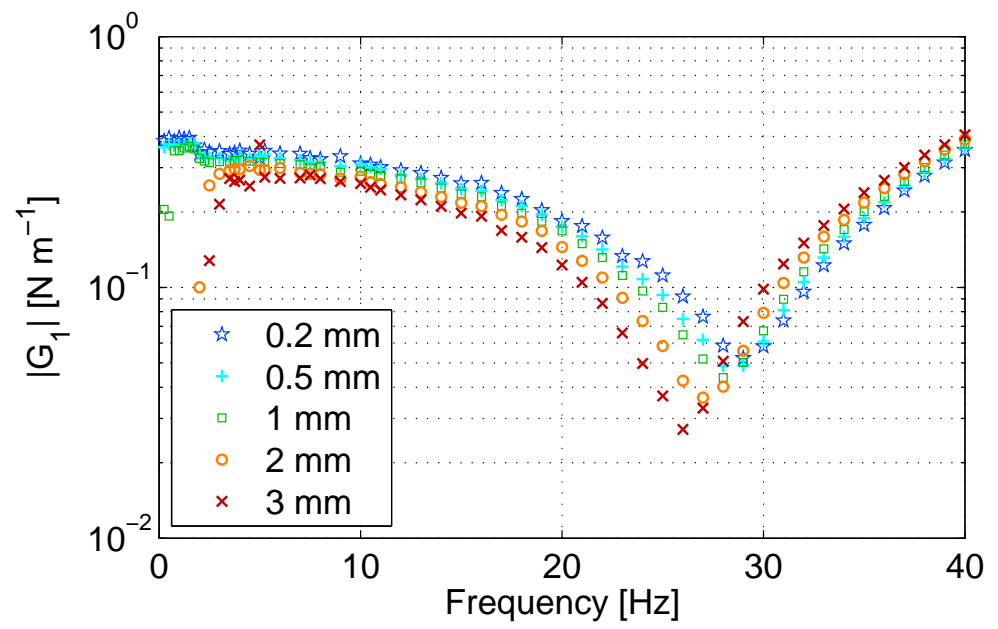

Figure 3. $\left|G_{1}(f)\right|$ for different values of $x_{\max }$.

\subsection{Stiffness $K_{n}(f)$ and damping $R_{n}(f)$}

The stiffness $K_{n}(f)$ and damping $R_{n}(f)$ can be deduced from the estimated filters $G_{n}(f)$ using Eqs. (8-9), for each input level. $K_{1}(f)$ and $R_{1}(f)$ respectively describe linear contributions of stiffness and damping, while $K_{n}(f)$ and $R_{n}(f)$ (for $n \geq 2$ ) code their higher-order nonlinear contributions. Fig. 4 shows $K_{1}(f)$ and $R_{1}(f)$ for different values of $x_{\max }$. Except very low frequencies, where the results are affected by the noise, the stiffness $K_{1}(f)$ (Fig. 4(a)) may by considered as quasi-constant with frequency for any value of $x_{\max }$.

Indeed, the stiffness of the mechanical part of an electrodynamic loudspeaker is often considered to be frequency dependent. An increasing value of stiffness (or a decreasing value of compliance) with increasing frequency has been reported by several authors $[9,31,34,35]$. However, in these works, the variation of stiffness with frequency is observed for measurements for which the amplitude of the voltage excitation is kept constant. In such measurements, however, the displacement drops with increasing frequency as a natural consequence of voltage driven loudspeakers. On the other hand, the measurements provided in these experiments are conducted for a constant amplitude of the displacement. Moreover, it appears from Fig. $4(\mathrm{a})$ that $K_{1}(f)$ decreases when $x_{\max }$ increases, whatever the frequency. The value of linear stiffness contribution $K_{1}(f)$ is constant with frequency and decreases with increasing amplitude. Consequently, for a loudspeaker driven by voltage, the amplitude of the displacement would decrease with increasing frequency leading to an increase of the stiffness. 


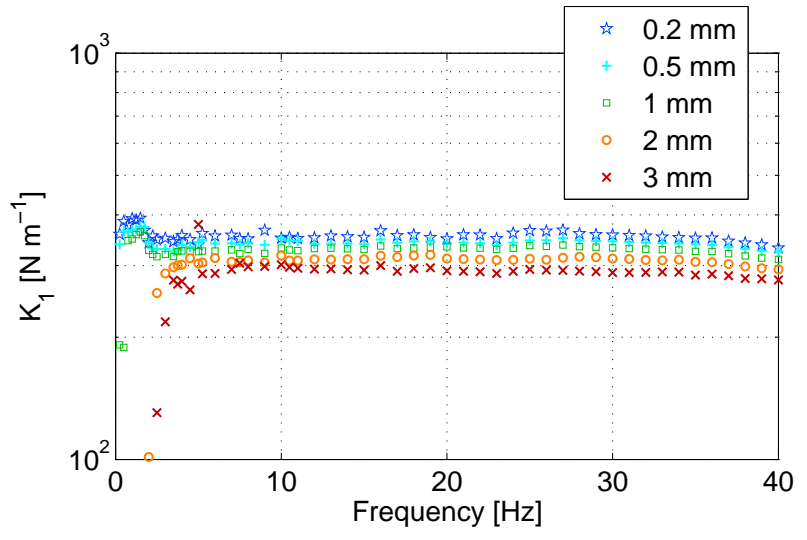

(a)

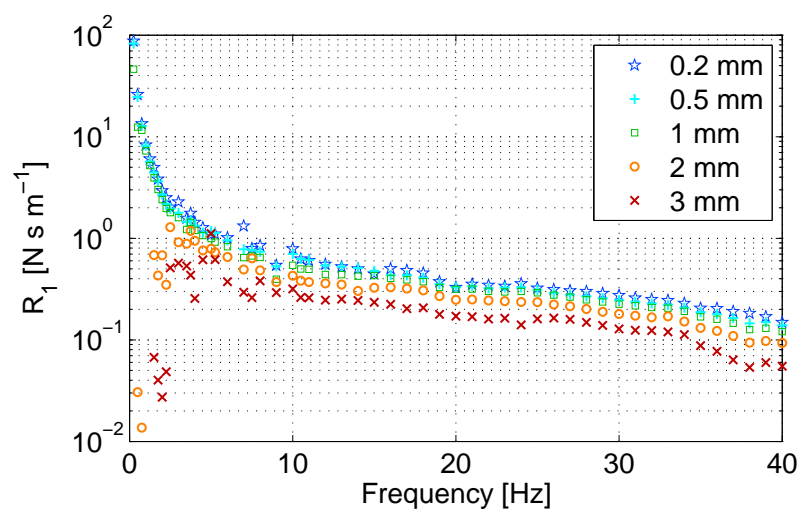

(b)

Figure 4. $K_{1}(f)(\mathrm{a})$ and $R_{1}(f)$ (b), for different values of $x_{\max }$. Loudspeaker : Visaton Sc8n.

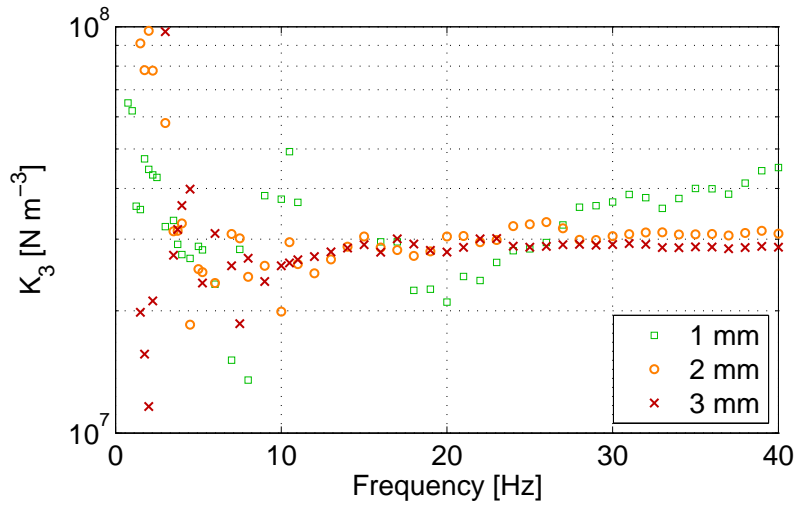

(a)

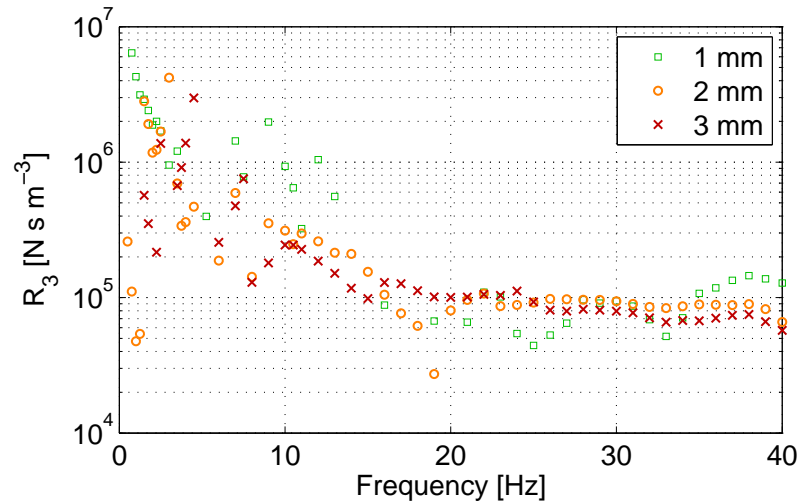

(b)

Figure 5. $K_{3}(f)(\mathrm{a})$ and $R_{3}(f)(\mathrm{b})$, for different values of $x_{\max }$. Loudspeaker : Visaton Sc8n.

For a given value of $x_{\max }$, the damping $R_{1}(f)$ (Fig. 4(b)) highlights a power law decreasing behaviour with frequency. Such a frequency dependence has already been observed and described by Thorborg et al. [9, 34], and is justified by two physical phenomenon; the viscous losses (frequency independent), and the elastic losses (decreasing when the frequency increases). Lastly, it appears that for all frequencies, $R_{1}(f)$ decreases when $x_{\max }$ increases.

The third-order stiffness $K_{3}(f)$ and damping $R_{3}(f)$ are depicted in Fig. 5 for the three highest amplitude levels $(1,2$, and $3 \mathrm{~mm})$. Lower levels, as well as higher or even orders, are not depicted due to low signal-to-noise ratio (SNR). The problematic of noise influence on the higher order kernels is detailed in section 6 . Both the stiffness $K_{3}(f)$ and the damping $R_{3}(f)$ show very similar behaviour as the linear contributions $K_{1}(f)$ and $R_{1}(f)$. The third-order stiffness $K_{3}(f)$ is almost constant, especially for levels $x_{\max }=2 \mathrm{~mm}$ and $x_{\max }=3 \mathrm{~mm}$ and within the frequency range $[10-40 \mathrm{~Hz}]$. Below $10 \mathrm{~Hz}$, the results are affected by the noise. While the frequency dependence of the third-order stiffness $K_{3}(f)$ and damping $R_{3}(f)$ behaves similarly compared to the linear stiffness $K_{1}(f)$ and damping $R_{1}(f)$, the dependence with amplitude $x_{\max }$ is less obvious to interpret for the third-order stiffness $K_{3}(f)$ and damping $R_{3}(f)$.

\subsection{Polynomial expressions of stiffness and resistance}

In the frequency domain, it has been shown that the mechanical behaviour of the loudspeaker moving part, represented by $K_{n}$ and $R_{n}$, depends on both frequency $f$ and driving amplitude $x_{\max }$. In order to describe this behaviour 
in the time domain, the following mathematical procedure is applied.

The coefficients $k_{n}$ and $r_{n}$ of the polynomial expansions of mechanical stiffness $K_{m s} \equiv k\{x(t)\}$

$$
k\{x(t)\}=\sum_{n=1}^{N} k_{n} \cdot x^{n-1}(t),
$$

and damping $R_{m s} \equiv r\{x(t)\}$

$$
r\{x(t)\}=\sum_{n=1}^{N} r_{n} \cdot x^{n-1}(t) .
$$

are estimated from the stiffness $K_{n}(f)$ and damping $R_{n}(f)$, as detailed in A.2. Coefficients $k_{n}$ and $r_{n}$ contribute to the time domain description of the mechanical behaviour of the loudspeaker moving part at driving frequency $f$.

Knowing the coefficients $k_{n}$, polynomials $k\{x(t)\}$ are then calculated for each input frequency and for each input level from Eq. 10. Polynomials $k\{x(t)\}$ are presented in Fig. 6 for the frequencies $5 \mathrm{~Hz}, 10 \mathrm{~Hz}$, and $15 \mathrm{~Hz}$, and for $\mathrm{N}=7$. On the one hand, these results highlight a stiffening of the moving part when $x(t)$ increases, and
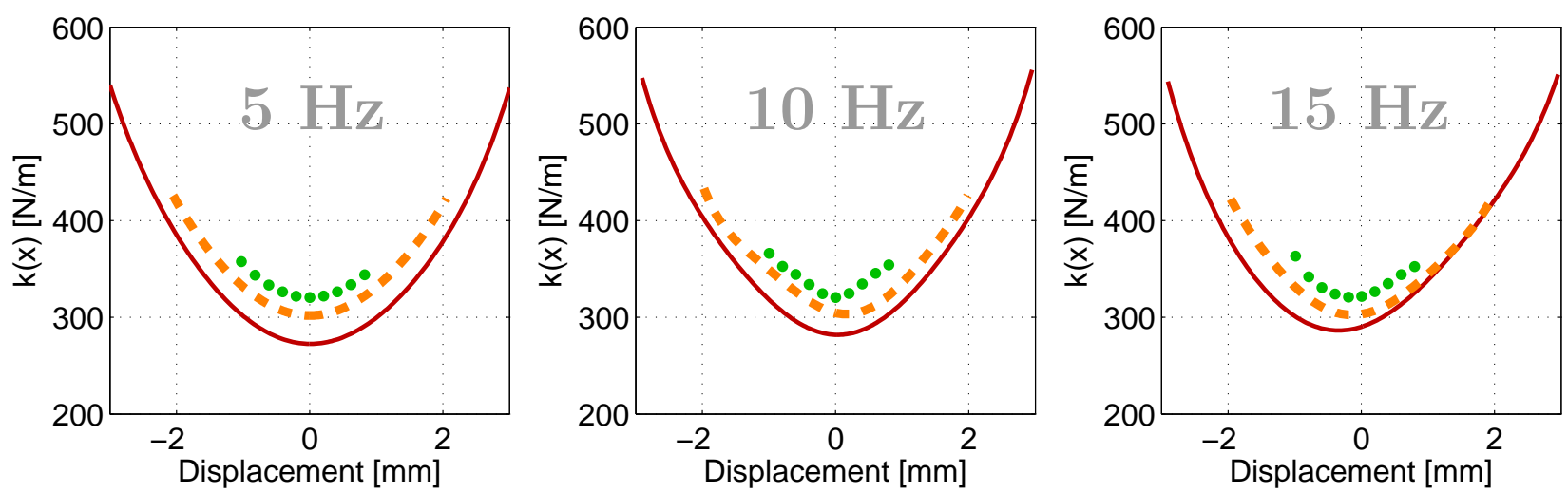

Figure 6. Polynomials $k\{x(t)\}$ based on estimated $k_{n}$ parameters $(n \in[1,7])$, for 3 input levels and 3 frequencies $f_{0}$, depicted for amplitudes $3 \mathrm{~mm}$ (red), $2 \mathrm{~mm}$ (orange), and $1 \mathrm{~mm}$ (green).
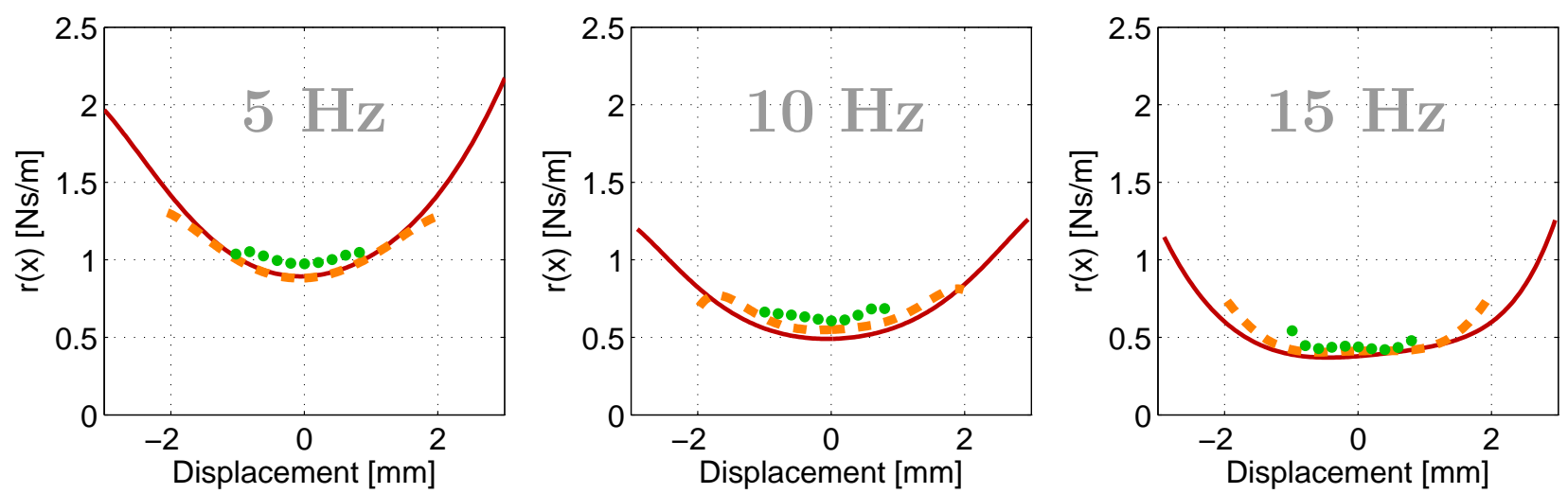

Figure 7. Polynomials $r\{x(t)\}$ based on estimated $r_{n}$ parameters $(n \in[1,7])$, for 3 input levels and 3 frequencies $f_{0}$, depicted for amplitudes $3 \mathrm{~mm}$ (red), $2 \mathrm{~mm}$ (orange), and $1 \mathrm{~mm}$ (green).

this is especially true for high values of $x_{\max }$, i.e. 2 or $3 \mathrm{~mm}$. This is in agreement with the variations of stiffness $K_{m s}(x)$ that is usually represented as a static polynomial [25]. On the other hand, $k\{x=0\}$ decreases when $x_{\text {max }}$ is increasing and consequently $k\{x(t)\}$ polynomials differ for each level. These observations are coherent with those of 
several authors $[25,26,35,36,37,38]$. Note also a small asymmetry of $k\{x(t)\}$ related to input frequency of $15 \mathrm{~Hz}$ (Fig. 6). This kind of asymmetry is created by even order of $k_{n}(n \in(2,4,6, \ldots))$ and, contrary to the asymmetry due to static offset of the voice coil rest position, this kind of asymmetry is created purely by the nonlinear dynamic behaviour.

The variation of the damping $r\{x(t)\}$ with instantaneous displacement $x(t)$ (Fig. 7) shows very similar behaviour.

\section{Discussion}

The nonlinear model proposed in this paper for describing the nonlinear viscoelastic behaviour of the mechanical part of an electrodynamic loudspeaker shows some interesting results. First, since the filter $G_{1}(f)$ varies with the input level, the moving part of a loudspeaker cannot be described by a unique input-independent Generalized Hammerstein model. Second, since the most common model describing the nonlinearity of the moving part of the loudspeaker and based on a static nonlinear stiffness $K_{m s}(x)$ is a subclass of the Generalized Hammerstein model used in this paper, the very same conclusion holds if the simple static nonlinear stiffness $K_{m s}(x)$ is used. Such a conclusion is not surprising. This kind of behaviour is well known for example in filled rubber isolators where for a single harmonic excitation they exhibit a strong amplitude dependence, where stiffness is high for small excitation amplitudes and low for large amplitudes [3]. Nevertheless, these well known properties are often neglected in loudspeaker suspension modelling.

We have furthermore shown that the linear stiffness $K_{1}(f)$ as well as the third order stiffness $K_{3}(f)$ remain almost constant with frequency and that the linear stiffness $K_{1}(f)$ depends slightly on the driving amplitude $x_{\max }$. The linear damping $R_{1}(f)$ and third-order damping $R_{3}(f)$ exhibit a similar power law as a function of frequency, and the value of $x_{\max }$ also influences the linear damping $R_{1}(f)$. These findings can be used in future works for real-time modelling and prediction of the loudspeaker behaviour. Since the frequency dependency seems to be very small for higher orders of $K_{n}$ and $R_{n}$, these might be simplified to constant values.

We have also noted that the higher order stiffness $K_{n}(f)$ and damping $R_{n}(f)$ are difficult to be measured due to the effect of noise. Indeed, SNR decreases when tested level decreases and $G_{n}(f)$ estimations consequently lead to a large variance for low levels. Likewise, because of the $G_{n}(f)$ estimation method, the noise has a greater effect for higher order $G_{n}(f)$ filters. This leads to a cautious use of higher order $G_{n}(f)$ filters, especially for low levels, and consequently of interpretation of $K_{n}(f)$ and $R_{n}(f)$. However, the values of $G_{1}(f)$ and of the linear contributions $K_{1}(f)$ and $R_{1}(f)$ are almost noise-free except for very low frequencies. Moreover, the higher orders are also involved into the calculation of the time domain nonlinear stiffness and resistance parameters $k_{n}$ and $r_{n}$. These parameters determine the $k\{x(t)\}$ and $r\{x(t)\}$ polynomials, respectively describing the stiffness and the resistance in time domain as a function of the instantaneous displacement $x(t)$. As depicted in Figs. 6 and 7 , the curves do not exhibit large noisy fluctuations even if the order $N=7$ was used.

While measuring and analysing the nonlinear effects of loudspeaker suspensions many precautions must be taken so that the estimated data are not influenced by other effect not related to the suspensions. A very important step is to get rid of all the nonlinearities generated by the devices used for the measurement, mainly the excitation device. In this paper, we use a technique developed in [32] in which a single harmonic excitation is used. Expanding the measurements to a multiple harmonic excitation or to other broadband signals would require a different technique allowing the separation of the nonlinear effects of the suspensions from the nonlinear effects of devices used for the measurement. Such a technique, being part of our perspectives, could reveal more details about the nonlinear properties of the loudspeaker suspension. A future work should also focus on the temperature dependence and the time-varying properties of the loudspeaker suspensions since there are both closely related to the nonlinear effects of the loudspeaker. Nevertheless, an emphasis should be placed on simplicity of the model so that it can be easily used by researchers and engineers from the loudspeaker industry. 


\section{Conclusion}

To sum up, the paper presents a model of loudspeaker suspensions that takes into account the nonlinear effects (usually modelled as a static nonlinearity of $K_{m s}$ ) as well as the viscoelastic behaviour (usually represented by frequency varying coefficients $K_{m s}$ (representing the stiffness) and $R_{m s}$ (representing the mechanical resistance)). While both concepts are well known, the models used for loudspeaker modelling take rarely into account both the viscoelastic effect (frequency dependent $K_{m s}$ and $R_{m s}$ ) and nonlinear behaviour.

The model and the measurement method presented in this paper show several interesting phenomena. First, from the nonlinear point of view, the estimated stiffness depends on the instantaneous displacement which is in agreement with the results found in the literature (stiffness increasing with positive and negative instantaneous displacement). Moreover, this behaviour seems to be dependent not only on the instantaneous displacement but also on the peak displacement as already pointed out in [26]. From the point of view of the frequency dependence of the viscoelastic parameters $K_{m s}$ and $R_{m s}$, an important frequency dependence of $R_{m s}$ has been found in agreement with the results in the literature. On the other hand, $K_{m s}$ has shown a very little variation with frequency in the studied frequency range contrary to what can be found in the literature. One of the possible explanation is that the variation of stiffness with frequency is often estimated from measurements for which the amplitude of the loudspeaker voltage excitation is kept constant. In such measurements, however, the displacement drops with increasing frequency as a natural consequence of voltage driven loudspeakers. On the other hand, the measurements provided in these experiments are conducted for a constant amplitude of the displacement. As shown in this paper the stiffness decreases when the peak displacement increases, whatever the frequency. The value of the linear contribution of the stiffness $K_{1}(f)$ is thus found to be independent of frequency but decreases with increasing amplitude. Consequently, the results found in this paper are in agreement with those found in the literature. For a loudspeaker driven by voltage, the amplitude of the displacement decreases with increasing frequency which, according to our findings, results in an increase of the stiffness.

Moreover, as shown in this paper, even if the considered model is a generalisation of existing models of loudspeaker suspensions, its parameters still vary with the amplitude of the peak displacement of the diaphragm. If one needs a precise model of the suspension (e.g. for a nonlinear compensator), these variations should be taken into account. 


\section{Appendix}

\section{A.1 Matrix for HHFRs}

Since the measurement technique described in this paper uses a shaker as the excitation device to create the displacement of the mechanical part of the loudspeaker under test, the nonlinearities caused by the shaker must be taken into account. The problem is depicted in Figure 8 in which the first NLS $\left(\mathrm{NL}_{1}\right)$ represents the shaker and the second NLS $\left(\mathrm{NL}_{2}\right)$ represents the mechanical part of the loudspeaker.

The excitation swept-sine signal $s(t)$ is the input of the first NLS. The displacement $x(t)$ is the output of the first NLS and the input of the second NLS. The force $f(t)$ is the output of the second NLS. Consequently, $f(t)$ may be seen as the output of the whole system for the input signal $s(t)$.

The method presented in [32] then allows the identification of the second NLS. The identification of the system is equivalent to estimating the linear filters $G_{n}(f), n=1, N$, from the measured signals $x(t)$ and $f(t)$. The identification process is based on the off-line estimation of both the Higher Harmonic Frequency Responses (HHFRs) $\mathcal{H}_{m}^{(x, s)}(f)$ between $s(t)$ and $x(t)$, and the HHFRs $\mathcal{H}_{m}^{(f, s)}(f)$ between $f(t)$ and $u(t)$, for $m=1, M, M$ being the total number of harmonics taken into account. We recall that, given an input signal $a(t)$ and an output signal $b(t)$ of a NLS, the HHFR $\mathcal{H}_{m}^{(b, a)}(f)$ may be seen as the contribution, in both amplitude and phase, of the $m$-th harmonic at the output, for a sine at frequency $f$ at the input, as

$$
\mathcal{H}_{m}^{(b, a)}(f)=\left|\mathcal{H}_{m}^{(b, a)}(f)\right| \mathrm{e}^{j \varphi_{m}^{(b, a)}(f)} .
$$

First, the HHFRs $\mathcal{H}_{m}^{(x, s)}(f)$ and $\mathcal{H}_{m}^{(f, s)}(f)$ are estimated using the synchronized swept sine method (for more details see [32] and [39]). Next, the displacement signal $x(t)$, already distorted by the shaker $\left(\mathrm{NL}_{1}\right)$, is taken to the powers of $n$ and HHFRs $\mathcal{H}_{m}^{\left(x^{n}, s\right)}(f)$ for $n=1, N$ are calculated.

The HHFRs $\mathcal{H}_{m}^{(f, s)}(f)$ of the output signal $f(t)$ result in the combination of all HHFRs $\mathcal{H}_{m}^{\left(x^{n}, s\right)}(f)$ after filtering by filters $G_{n}(f)$. The relation between the HHFRs $\mathcal{H}_{m}^{\left(x^{n}, s\right)}(f), \mathcal{H}_{m}^{(f, s)}(f)$ and the linear filters $G_{n}(f)$ can indeed be written in a matrix form as [32]

$$
\begin{aligned}
{\left[\begin{array}{c}
\mathcal{H}_{1}^{(f, s)}(f) \\
\mathcal{H}_{2}^{(f, s)}(f) \\
\vdots \\
\mathcal{H}_{M}^{(f, s)}(f)
\end{array}\right]=} & {\left[\begin{array}{cccc}
\mathcal{H}_{1}^{(x, s)}(f) & \mathcal{H}_{1}^{\left(x^{2}, s\right)}(f) & \ldots & \mathcal{H}_{1}^{\left(x^{N}, s\right)}(f) \\
\mathcal{H}_{2}^{(x, s)}(f) & \mathcal{H}_{2}^{\left(x^{2}, s\right)}(f) & \ldots & \mathcal{H}_{2}^{\left(x^{N}, s\right)}(f) \\
\vdots & \vdots & \ddots & \vdots \\
\mathcal{H}_{M}^{(x, s)}(f) & \mathcal{H}_{M}^{\left(x^{2}, s\right)}(f) & \cdots & \mathcal{H}_{M}^{\left(x^{N}, s\right)}(f)
\end{array}\right] } \\
& \times\left[\begin{array}{c}
G_{1}(f) \\
G_{2}(f) \\
\vdots \\
G_{N}(f)
\end{array}\right] .
\end{aligned}
$$

Equation (A.2) can then be solved for unknown $G_{n}(f)$ by using a square matrix inversion in the case $M=N$, or by using a pseudo-inversion in the case $M>N$. The number of harmonics $M$ chosen for the estimation of HHFRs must be equal or greater than the number of the branches $N$ of the Generalized Hammerstein model. The matrix (pseudo)-inversion must be computed for each frequency $f$ separately. 


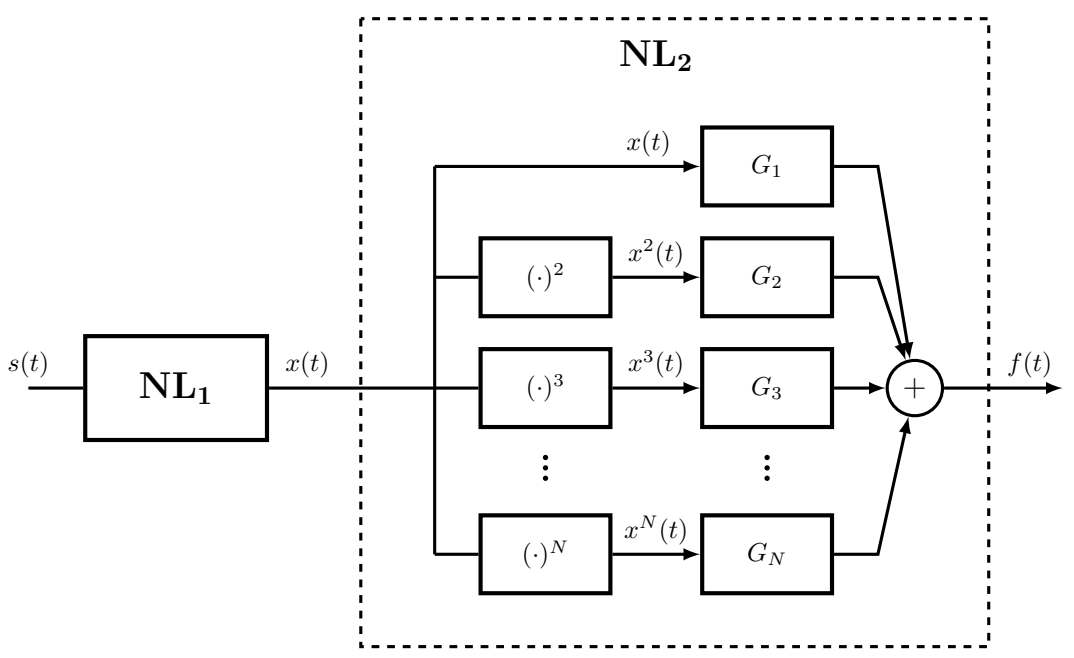

Figure 8. Two dynamic nonlinear systems in series, the first one represents the shaker, the second one, represented by a Generalized Hammerstein model, is the mechanical part of the loudspeaker under test.

\section{A.2 Time-domain coefficients $k_{n}$ and $r_{n}$}

We first replace $K_{m s}$ of Eq. (10) and $R_{m s}$ of Eq. (11) in the equation of movement Eq. (1). We then use Eq. (4-5) for expressing the equation in the Fourier domain, which leads to

$$
F(f)=(\mathrm{i} 2 \pi f)^{2} M_{m s} X(f)+\sum_{n=1}^{N}\left(\left(\frac{\mathrm{i} 2 \pi f r_{n}}{n}+k_{n}\right) X^{(n)}(f)\right),
$$

with $X^{(n)}(f)$ the Fourier transform of $x^{n}(t)$. In Eq. (A.3), we suppose that both $r_{n}$ and $k_{n}$ depend on frequency $f$. We respectively note them $R_{n}(f)$ and $K_{m}(f)$ and we finally have

$$
F(f)=(\mathrm{i} 2 \pi f)^{2} M_{m s} X(f)+\sum_{n=1}^{N}\left[\left(\frac{\mathrm{i} 2 \pi f R_{n}(f)}{n}+K_{n}(f)\right) X^{(n)}(f)\right]
$$

with $R_{n}(f), K_{n}(f) \in \mathbb{R}, \forall n \in[1, N], \forall f$. This equation now has to be compared with the equation of the Generalized Hammerstein Model Eq. (7). For doing this, we suppose that the input of the system is $x(t)=X_{0} \cos \left(2 \pi f_{0} t\right)$.

In the time domain, we then have

$$
F(t)=-M\left(2 \pi f_{0}\right)^{2} x(t)+\sum_{\substack{n=2 \\
n \text { even }}}^{N} \frac{X_{0}^{n}}{2^{n}}\left(\begin{array}{c}
n \\
n / 2
\end{array}\right) K_{n}(0)+I_{1}+I_{2}+I_{3}+I_{4},
$$

the integrals $I_{k}$ checking

$$
\left\{\begin{array}{l}
I_{1}=\sum_{\substack{n=1 \\
n \text { odd }}}^{N} \frac{X_{0}^{n}}{2^{n-1}} \sum_{p=0}^{\frac{n-1}{2}}\left(\begin{array}{c}
n \\
p+\frac{n+1}{2}
\end{array}\right) K_{n}\left((2 p+1) f_{0}\right) \frac{2 p+1}{2} \sum_{k=0}^{p}(-1)^{k} 2^{2(p-k)+1} \frac{(2 p-k) !}{k !(2(p-k)+1) !}(x(t))^{2(p-k)+1}, \\
I_{2}=\sum_{\substack{n=2 \\
n \text { even }}}^{N} \frac{X_{0}^{n}}{2^{n-1}} \sum_{p=1}^{\frac{n}{2}}\left(\begin{array}{c}
n \\
p+\frac{n}{2}
\end{array}\right) K_{n}\left(2 p f_{0}\right) p \sum_{k=0}^{p}(-1)^{k} 2^{2(p-k)} \frac{(2 p-k-1) !}{k !(2(p-k)) !}(x(t))^{2(p-k)} \\
I_{3}=\sum_{\substack{n=1 \\
n \text { odd }}}^{N} \frac{X_{0}^{n}}{n 2^{n-1}} \sum_{p=0}^{\frac{n-1}{2}}\left(\begin{array}{c}
n \\
p+\frac{n+1}{2}
\end{array}\right) R_{n}\left((2 p+1) f_{0}\right) \frac{2 p+1}{2} \sum_{k=0}^{p}(-1)^{k} 2^{2(p-k)+1} \frac{(2 p-k) !}{k !(2(p-k)+1) !} \frac{\mathrm{d}}{\mathrm{d} t}\left[(x(t))^{2(p-k)+1}\right], \\
I_{4}=\sum_{\substack{n=2 \\
n \text { even }}}^{N} \frac{n X_{0}^{n}}{2^{n-1}} \sum_{p=1}^{\frac{n}{2}}\left(\begin{array}{c}
n \\
p+\frac{n}{2}
\end{array}\right) R_{n}\left(2 p f_{0}\right) p \sum_{k=0}^{p}(-1)^{k} 2^{2(p-k)} \frac{(2 p-k-1) !}{k !(2(p-k)) !} \frac{\mathrm{d}}{\mathrm{d} t}\left[(x(t))^{2(p-k)}\right] .
\end{array}\right.
$$


By

1. noting $n=2 n^{\prime}+1$ (odd cases) and $n=2 n^{\prime}$ (even cases), $N_{1}=(N-1) / 2\left(N\right.$ odd) and $N_{1}=N / 2-1(N$ even), $N_{2}=(N-1) / 2(N$ odd $)$ and $N_{2}=N / 2(N$ even $)$,

2. replacing $\sum_{n^{\prime}=0}^{N_{1}} \sum_{p=0}^{n^{\prime}}$ by $\sum_{p=0}^{N_{1}} \sum_{n^{\prime}=p}^{N_{1}}$ (odd cases), $\sum_{n^{\prime}=1}^{N_{2}} \sum_{p=1}^{n^{\prime}}$ by $\sum_{p=1}^{N_{2}} \sum_{n^{\prime}=p}^{N_{2}}$ (even cases),

3. replacing $\sum_{p=0}^{N_{1}} \sum_{k=0}^{p}$ by $\sum_{r^{\prime}=0}^{N_{1}} \sum_{p=r^{\prime}}^{N_{1}}$ (odd cases), $\sum_{p=1}^{N_{2}} \sum_{k=0}^{p}$ by $\sum_{r^{\prime}=0}^{N_{2}} \sum_{p=\max \left(r^{\prime}, 1\right)}^{N_{2}}$ (even cases), we then have

$$
\begin{aligned}
& I_{1}=\sum_{r^{\prime}=0}^{N_{1}}\left(\sum_{p=r^{\prime}}^{N_{1}} \sum_{n^{\prime}=p}^{N_{1}} \frac{X_{0}^{2 n^{\prime}+1}}{2^{2 n^{\prime}}}\left(\begin{array}{c}
2 n^{\prime}+1 \\
p+n^{\prime}+1
\end{array}\right) K_{2 n^{\prime}+1}\left((2 p+1) f_{0}\right) \frac{2 p+1}{2}(-1)^{p-r^{\prime}} \frac{\left(p+r^{\prime}\right) !}{\left(p-r^{\prime}\right) !\left(2 r^{\prime}+1\right) !}\right) 2^{2 r^{\prime}+1}(x(t))^{2 r^{\prime}+1}, \\
& I_{2}=\sum_{r^{\prime}=0}^{N_{2}}\left(\sum_{p=\max \left(r^{\prime}, 1\right)}^{N_{2}} \sum_{n^{\prime}=p}^{N_{2}} \frac{X_{0}^{2 n^{\prime}}}{2^{2 n^{\prime}-1}}\left(\begin{array}{c}
2 n^{\prime} \\
p+n^{\prime}
\end{array}\right) K_{2 n^{\prime}}\left(2 p f_{0}\right) p(-1)^{p-r^{\prime}} \frac{\left(p+r^{\prime}-1\right) !}{\left(p-r^{\prime}\right) !\left(2 r^{\prime}\right) !}\right) 2^{2 r^{\prime}}(x(t))^{2 r^{\prime}} \text {, } \\
& I_{3}=\sum_{r^{\prime}=0}^{N_{1}}\left(\sum_{p=r}^{N_{1}} \sum_{n^{\prime}=p}^{N_{1}} \frac{X_{0}^{2 n^{\prime}+1}}{\left(2 n^{\prime}+1\right) 2^{2 n^{\prime}}}\left(\begin{array}{c}
2 n^{\prime}+1 \\
p+n^{\prime}+1
\end{array}\right) R_{2 n^{\prime}+1}\left((2 p+1) f_{0}\right) \frac{2 p+1}{2}(-1)^{p-r^{\prime}} \frac{\left(p+r^{\prime}\right) !}{\left(p-r^{\prime}\right) !\left(2 r^{\prime}+1\right) !}\right) 2^{2 r^{\prime}+1} \frac{\mathrm{d}}{\mathrm{d} t}\left[(x(t))^{2 r^{\prime}+1}\right] \text {, } \\
& I_{4}=\sum_{r^{\prime}=0}^{N_{2}}\left(\sum_{p=\max \left(r^{\prime}, 1\right)}^{N_{2}} \sum_{n^{\prime}=p}^{N_{2}} \frac{X_{0}^{2 n^{\prime}}}{2 n^{\prime} 2^{2 n^{\prime}-1}}\left(\begin{array}{c}
2 n^{\prime} \\
p+n^{\prime}
\end{array}\right) R_{2 n^{\prime}}\left(2 p f_{0}\right) p(-1)^{p-r^{\prime}} \frac{\left(p+r^{\prime}-1\right) !}{\left(p-r^{\prime}\right) !\left(2 r^{\prime}\right) !}\right) 2^{2 r^{\prime}} \frac{\mathrm{d}}{\mathrm{d} t}\left[(x(t))^{2 r^{\prime}}\right] .
\end{aligned}
$$

Consequently, the coefficients $k_{n}$ and $r_{n}$ of the polynomial expansions of $K_{m s}$ and $R_{m s}$ check, for $r^{\prime} \in\left[1, N_{1}\right]$ (odd cases) or for $r^{\prime} \in\left[1, N_{2}\right]$ (even cases),

$$
\left\{\begin{array}{l}
k_{2 r^{\prime}+1}=\sum_{p=r^{\prime}}^{N_{1}} \frac{2 p+1}{2}(-1)^{p-r^{\prime}} \frac{\left(p+r^{\prime}\right) !}{\left(p-r^{\prime}\right) !\left(2 r^{\prime}+1\right) !} \sum_{n^{\prime}=p}^{N_{1}} 2^{2\left(r^{\prime}-n^{\prime}\right)+1} \frac{\left(2 n^{\prime}+1\right) !}{\left(p+n^{\prime}+1\right) !\left(n^{\prime}-p\right) !} X_{0}^{2 n^{\prime}+1} K_{2 n^{\prime}+1}\left((2 p+1) f_{0}\right), \\
k_{2 r^{\prime}}=\sum_{p=\max \left(r^{\prime}, 1\right)}^{N_{2}} p(-1)^{p-r^{\prime}} \frac{\left(p+r^{\prime}-1\right) !}{\left(p-r^{\prime}\right) !\left(2 r^{\prime}\right) !} \sum_{n^{\prime}=p}^{N_{2}} 2^{2\left(r^{\prime}-n^{\prime}\right)+1} \frac{\left(2 n^{\prime}\right) !}{\left(p+n^{\prime}\right) !\left(n^{\prime}-p\right) !} X_{0}^{2 n^{\prime}} K_{2 n^{\prime}}\left(2 p f_{0}\right), \\
r_{2 r^{\prime}+1}=\sum_{p=r_{1}^{\prime}}^{N_{1}} \frac{2 p+1}{2}(-1)^{p-r^{\prime}} \frac{\left(p+r^{\prime}\right) !}{\left(p-r^{\prime}\right) !\left(2 r^{\prime}+1\right) !} \sum_{n^{\prime}=p}^{N_{1}} 2^{2\left(r^{\prime}-n^{\prime}\right)+1} \frac{2 r^{\prime}+1}{2 n^{\prime}+1} \frac{\left(2 n^{\prime}+1\right) !}{\left(p+n^{\prime}+1\right) !\left(n^{\prime}-p\right) !} X_{0}^{2 n^{\prime}+1} R_{2 n^{\prime}+1}\left((2 p+1) f_{0}\right), \\
r_{2 r^{\prime}}=\sum_{p=\max \left(r^{\prime}, 1\right)}^{N_{2}} p(-1)^{p-r^{\prime}} \frac{\left(p+r^{\prime}-1\right) !}{\left(p-r^{\prime}\right) !\left(2 r^{\prime}\right) !} \sum_{n^{\prime}=p}^{N_{2}} 2^{2\left(r^{\prime}-n^{\prime}\right)+1} \frac{r^{\prime}}{n^{\prime}} \frac{\left(2 n^{\prime}\right) !}{\left(p+n^{\prime}\right) !\left(n^{\prime}-p\right) !} X_{0}^{2 n^{\prime}} R_{2 n^{\prime}}\left(2 p f_{0}\right) .
\end{array}\right.
$$




\section{References}

[1] G. Kerschen, K. Worden, A. F. Vakakis, J.-C. Golinval, Past, present and future of nonlinear system identification in structural dynamics, Mech. Syst. Signal Process. 20 (3) (2006) 505-592.

[2] J.-P. Noël, G. Kerschen, Nonlinear system identification in structural dynamics: 10 more years of progress, Mech. Syst. Signal Process. 83 (2017) 2-35.

[3] M. Sjöberg, L. Kari, Testing of nonlinear interaction effects of sinusoidal and noise excitation on rubber isolator stiffness, Polym. Test. 22 (3) (2003) 343-351.

[4] F. M. Guillot, D. Trivett, Complete elastic characterization of viscoelastic materials by dynamic measurements of the complex bulk and young's moduli as a function of temperature and hydrostatic pressure, J. Sound Vib. 330 (14) (2011) 3334-3351.

[5] A. de Lima, D. Rade, H. Lacerda, C. Araújo, An investigation of the self-heating phenomenon in viscoelastic materials subjected to cyclic loadings accounting for prestress, Mech. Syst. Signal Process. 58 (2015) 115-127.

[6] Ž. Jelčić, V. O. Bulatović, V. Rek, K. J. Marković, Relationship between fractal, viscoelastic, and aging properties of linear and radial styrene-butadiene-styrene polymer-modified bitumen, J. Elastomers Plast. (2014) 0095244314538437.

[7] R. H. Small, Closed-box loudspeaker systems-part 1: analysis, J. Audio Eng. Soc 20 (10) (1972) 798-808.

[8] M. H. Knudsen, J. G. Jensen, Low-frequency loudspeaker models that include suspension creep, J. Audio Eng. Soc $41(1 / 2)(1993) 3-18$.

[9] K. Thorborg, C. Futtrup, Frequency dependence of the loudspeaker suspension (a follow up), J. Audio Eng. Soc 61 (10) (2013) 778-786.

[10] W. Klippel, Tutorial: Loudspeaker nonlinearities - causes, parameters, symptoms, J. Audio Eng. Soc 54 (10) (2006) 907-939.

[11] E. Wiechert, Gesetze der elastischen nachwirkung für constante temperatur, Annalen der Physik 286 (11) (1893) 546-570.

[12] K. Ege, X. Boutillon, M. Rébillat, Vibroacoustics of the piano soundboard:(non) linearity and modal properties in the low-and mid-frequency ranges, J. Sound Vib. 332 (5) (2013) 1288-1305.

[13] M. Rébillat, R. Hajrya, N. Mechbal, Nonlinear structural damage detection based on cascade of hammerstein models, Mech. Syst. Signal Process. 48 (1) (2014) 247-259.

[14] D. R. Morgan, Z. Ma, J. Kim, M. G. Zierdt, J. Pastalan, A generalized memory polynomial model for digital predistortion of rf power amplifiers, IEEE Trans. Signal Process. 54 (10) (2006) 3852-3860.

[15] A. Novak, L. Simon, P. Lotton, F. Kadlec, Nonlinear system identification using exponential swept-sine signal, IEEE Trans. Instrum. Meas. 59 (8) (2010) 2220-2229.

[16] M. Rebillat, R. Hennequin, E. Corteel, B. F. Katz, Identification of cascade of hammerstein models for the description of nonlinearities in vibrating devices, J. Sound Vib. 330 (5) (2011) 1018-1038.

[17] R. S. Risuleo, G. Bottegal, H. Hjalmarsson, A kernel-based approach to hammerstein system identification, IFAC-PapersOnLine 48 (28) (2015) 1011-1016. 
[18] A. J. M. Kaizer, Modeling of the nonlinear response of an electrodynamic loudspeaker by a volterra series expansion, J. Audio Eng. Soc 35 (6) (1987) 421-433.

[19] N. Quaegebeur, A. Chaigne, Nonlinear vibrations of loudspeaker-like structures, J. Sound Vib. 309 (1) (2008) $178-196$.

[20] R. Mariani, S. Bellizzi, B. Cochelin, P. Herzog, P.-O. Mattei, Toward an adjustable nonlinear low frequency acoustic absorber, J. Sound Vib. 330 (22) (2011) 5245-5258.

[21] Z. Zhang, Q. Tao, Experimental study of non-linear vibrations in a loudspeaker cone, J. Sound Vib. 248 (1) (2001) $1-8$.

[22] T. Heed, Quantitative analysis of low-frequency component nonlinearities, in: 101th Convention of the Audio Engineering Society, 1996.

[23] S. Hutt, Loudspeaker spider linearity, in: 106th Convention of the Audio Engineering Society, 2000.

[24] A. Saraswat, N. Tiwari, Modeling and study of nonlinear effects in electrodynamic shakers, Mech. Syst. Signal Process. 85 (2017) 162-176.

[25] W. Klippel, Dynamic measurement of loudspeaker suspension parts, J. Audio Eng. Soc 55 (6) (2007) 443-459.

[26] A. Novak, P. Lotton, L. Simon, Dynamic measurement of loudspeaker suspension parameters using an active harmonic control technique, in: 136th Convention of the Audio Engineering Society, 2014.

[27] M. Lumori, J. Schoukens, J. Lataire, Identification and quantification of nonlinear stiffness and nonlinear damping in resonant circuits, Mech. Syst. Signal Process. 24 (7) (2010) 1938-1949.

[28] M. Zilletti, A. Marker, S. J. Elliott, K. Holland, Identifying the nonlinear mechanical behaviour of microspeakers from their quasi-linear electrical response, Mech. Syst. Signal Process. 88 (2017) 212-223.

[29] K. Satoh, H. Takewa, M. Iwasa, The measuring method of dynamic force-to-displacement characteristics for loudspeaker suspension system and driving force, in: 107th Convention of the Audio Engineering Society, 1999.

[30] M. Rousseau, J. Vanderkooy, Visco-elastic aspects of loudspeaker drivers, in: 118th Convention of the Audio Engineering Society, Barcelona, Spain, 2005.

[31] A. Novak, Modeling viscoelastic properties of loudspeaker suspensions using fractional derivatives, J. Audio Eng. Soc $64(1 / 2)(2016) 35-44$.

[32] A. Novak, B. Maillou, P. Lotton, L. Simon, Nonparametric identification of nonlinear systems in series, IEEE Trans. Instrum. Meas. 63 (8) (2014) 2044-2051.

[33] W. Klippel, Distortion analyzer-a new tool for assessing and improving electrodynamic transducer, in: 108th Convention of the Audio Engineering Society, Audio Engineering Society, 2000.

[34] K. Thorborg, C. Tinggaard, F. Agerkvist, C. Futtrup, Frequency dependence of damping and compliance in loudspeaker suspensions, J. Audio Eng. Soc 58 (6) (2010) 472-486.

[35] F. Agerkvist, B. R. Pedersen, Time variance of the suspension nonlinearity, in: 125th Convention of the Audio Engineering Society, 2008.

[36] E. S. Olsen, Measurement of mechanical parameter nonlinearities of electrodynamic loudspeakers, in: 98th Convention of the Audio Engineering Society, Audio Engineering Society, 1995. 
[37] F. Agerkvist, Modelling loudspeaker non-linearities, in: 32nd International Conference of the Audio Engineering Society: DSP For Loudspeakers, 2007.

[38] B. R. Pedersen, F. T. Agerkvist, Time varying behavior of the loudspeaker suspension, in: 123th Convention of the Audio Engineering Society, 2007.

[39] A. Novak, P. Lotton, L. Simon, Synchronized swept-sine: Theory, application, and implementation, J. Audio Eng. Soc 63 (10) (2015) 786-798. 